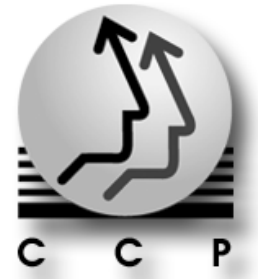

Población y Salud en Mesoamérica

Revista electrónica publicada por el

Centro Centroamericano de Población,

Universidad de Costa Rica, 2060 San José, Costa Rica

http://ccp.ucr.ac.cr

Población y Salud en Mesoamérica

Revista electrónica semestral, ISSN-1659-0201

Volumen 9, número 1 , artículo 5

Julio - diciembre, 2011

Publicado 1 de julio, 2011

http://ccp.ucr.ac.cr/revista/

\title{
Estudio de factores predisponentes de enfermedad diarreica aguda en la comunidad de San Simón, Yucatán en base a un análisis de vulnerabilidad nutricional y ambiental
}

\author{
Mariel Gullian-Klanian \\ Janice Guadalupe Durán-Casanova \\ María Luisa Isla-Esquivel \\ Estefanía Suárez-Wegan \\ Alberto Alarcón-Sánchez
}

(cc) BY-NO-ND

Protegido bajo licencia Creative Commons

Centro Centroamericano de Población 


\title{
Estudio de factores predisponentes de enfermedad diarreica aguda en la comunidad de San Simón, Yucatán en base a un análisis de vulnerabilidad nutricional y ambiental
}

\author{
Study of predisposing factors for acute diarrheal disease in the community of San Simon, \\ Yucatán based on environmental and nutritional vulnerability analysis
}

\author{
Mariel Gullian-Klanian, Janice Guadalupe Durán-Casanova, María Luisa Isla-Esquivel, Estefanía \\ Suárez-Wegan, Alberto Alarcón-Sánchez ${ }^{1}$
}

\section{RESUMEN}

Durante el periodo 2005-2008, 32.1\% de los residentes de la comunidad de San Simón padecieron de enfermedad diarreica aguda (EDA). Durante este periodo la población más afectada fue la infantil, específicamente los niños menores de 10 años de los cuales $40 \%$ se encontraban en diferente rango de desnutrición. Objetivo: Identificar factores predisponentes de enfermedad diarreica aguda (EDA) de origen bacteriano en San Simón en base a un análisis de vulnerabilidad (AV) nutricional y ambiental. Metodología: La descripción socio-demográfica y ambiental de la comunidad se realizó en base a datos obtenidos de un censo poblacional. Los datos del censo se agruparon en 2 indicadores: indicadores de demanda de servicios (D) e indicadores de oferta de servicios (O) los cuales sirvieron para calcular el índice de vulnerabilidad de la comunidad $[\mathrm{AV}(\mathrm{AV}=\mathrm{D} / \mathrm{S})]$. Mediante indicadores antropométricos se determinó la vulnerabilidad nutricional en 106 personas. Como factor indicador de EDA se realizó el diagnóstico microbiológico y serológico de Escherichia coli 0157:H7 y Salmonella spp. en hortalizas (n=45) y animales portadores $(\mathrm{n}=64)$. La presencia de estas bacterias se tomó como factor de amenaza. La valoración del riesgo de la población de padecer EDA se consideró teniendo en cuenta la predisposición hacia la amenaza y su efecto sobre la salud de la población. Resultados: El estado nutricional, el porcentaje de morbilidad y el bajo nivel de educación, fueron los indicadores de mayor peso en el AV. Se presentaron $43.2 \%$ de casos de desnutrición grave en niños. El $60.6 \%$ de las mujeres y el $53.5 \%$ de los hombres presentaron obesidad. Respecto al riesgo, $80.6 \%$ de los cerdos fueron portadores de E. coli $\mathrm{O} 157: \mathrm{H} 7$. El $35.7 \%$ de las aves y $8.3 \%$ de las hortalizas presentaron Salmonella spp. Conclusiones: San Simón presenta un riesgo ambiental alto $(\mathrm{R}=39 \pm 10.6)$ y una vulnerabilidad intermedia $(\mathrm{V}=4.9)$ de padecer EDA.

Palabras claves: Vulnerabilidad nutricional y ambiental; Enfermedad diarreica aguda; Yucatán, México

\begin{abstract}
During 2005-2008, 32.1\% of the population from San Simon (Yucatán, México) was diagnosed with acute diarrheal disease (ADD). During this period the population most affected was children under 10 years of which $40 \%$ have different range of malnutrition. Objective. Identify predisposing factors of acute diarrheal disease (ADD) in the San Simon community, based on a nutritional and environmental vulnerability analysis (VA). Material and methods. A population census was carried out focusing on socio-cultural, economic and health aspects to identify indicators of demand (D) and supply (S) as components of the VA $(\mathrm{VA}=\mathrm{D} / \mathrm{S})$. The threat assessment was determined taking into account the predisposition to the risk factor and its effect on population health. The risk (R) was calculated multiplying the vulnerability index by the threat value. The nutritional vulnerability was determined using anthropometric indicators $(\mathrm{n}=106)$. The presence of threat factor $(\mathrm{TF})$ represented by Escherichia coli O157: H7 and Salmonella spp. was determined on vegetables $(n=45)$ and animal feces $(n=64)$. Results. The nutritional disorders, the morbidity rate and the low educational level were the main indicators in the vulnerability analysis. There were $43.2 \%$ children with severe malnutrition, $60.6 \%$ of women and $53.5 \%$ of men with obesity. Regarding the study of the risk, $80.6 \%$ of the pigs were E. coli O157: H7 positive. Salmonella spp. was found in $35.7 \%$ of chicken and $8.3 \%$ of vegetables. Conclusion. The population of San Simon has a high environmental risk $(\mathrm{R}=39 \pm 10.6)$ and intermediate vulnerability $(\mathrm{V}=4.9)$ of suffering EDA.
\end{abstract}

Keywords: Nutritional and environmental vulnerability; Acute diarrheal disease; Yucatán, México

\footnotetext{
${ }^{1}$ Universidad Marista de Mérida, Yucatán.mgullian@marista.edu.mx, janicita_0306@hotmail.com, misla@marista.edu.mx, esuarez@marista.edu.mx, aalarcon@marista.edu.mx, MÉXICO
} 


\section{INTRODUCCIÓN}

A lo largo del territorio mexicano existe una gran variedad de comunidades indígenas que definen la existencia de una rica conformación étnica. Dichas comunidades se concentran principalmente en la Sierra Madre del Sur, la Península de Yucatán y en la Sierra Madre Oriental y Occidental. En estas comunidades, la posibilidad de un desarrollo económico propio y autónomo muchas veces se ve limitado por factores como la segregación y el analfabetismo.

La comunidad maya de San Simón está ubicada en el municipio de Santa Elena, al suroeste del estado de Yucatán (2009“y 20o 24“ N, 89o 36“y 89o 55“ O). Aunque se encuentra a escasos 34 $\mathrm{km}$ de la cabecera municipal de Sta. Elena y a $95 \mathrm{~km}$ de la ciudad de Mérida, capital del estado de Yucatán, continúa siendo una comunidad con alto nivel de marginación debido al acceso limitado a los servicios de salud y alimentos, al rezago educativo promedio y al acceso a los servicios básicos de vivienda (CONEVAL, 2005).

A finales del siglo XVIII, San Simón fue una de las tantas haciendas que existían en el estado. Actualmente la Hacienda de San Simón está completamente en ruinas y los pobladores que conforman la comunidad, son descendientes de los antiguos trabajadores que se encontraban a las órdenes de los hacendados. La comunidad de San Simón no cuenta con un medio de transporte público, lo que le impide su inserción en la vida del municipio. La única vía de acceso es a través de una carretera sinuosa de un carril de $18 \mathrm{~km}$ que comunica a esta comunidad con la zona arqueológica de Uxmal. Al sur existe un camino blanco o $S a c$ be de $3 \mathrm{~km}$ de longitud, por el cual los habitantes de San Simón llegan a pie a la comunidad de X'culoc (su significado es "sin pies") comisaría del municipio de Hopelchén, en el estado vecino de Campeche. Si bien, la comunidad cuenta con una caseta de teléfono satelital de tipo rural, la señal no es nítida debido a su ubicación entre cerros. Tampoco existen oficinas de servicio postal y una patrulla policial se encarga de realizar la vigilancia, cumpliendo el rol de ambulancia y mensajería cuando es necesario, y comunicándose por medio de radio con otras localidades vecinas. La atención a la salud pública se ofrece a través de una pequeña clínica, la cual cuenta con área de consultorio, sala de espera y un cuarto médico. El personal está integrado por un médico y una enfermera de ocasión temporal y un promotor de salud cuya función es llevar a cabo pláticas y programas de salud para la comunidad.

Su condición de marginalidad hace a esta comunidad vulnerable a diversos factores dentro de los cuales podemos mencionar su condición nutricional debido al limitado acceso a alimentos de alto valor nutricional y la exposición a patógenos ambientales a causa del escaso saneamiento ambiental y el inadecuado manejo de animales de su propia crianza. La contaminación ambiental por mal manejo de residuos y malas prácticas en la crianza de animales representa un riesgo hacia el ser humano, más aún si la población es considerada vulnerable. En comunidades carentes de sistemas de saneamiento, es altamente probable el reciclaje de patógenos y su diseminación, especialmente de bacterias enteropatógenas como Escherichia coli O157:H7 y Salmonella spp., constituyéndose en factores de riesgo (Yates, 1992). Las principales fuentes de contaminación son el agua de riego y el estiércol de los animales sin embargo, dentro de su epidemiología intervienen multitud de factores interdependientes que facilitan la diseminación de modo directo o indirecto entre animales, suelo, hortalizas y el ser humano (Le Minor, 1992). 
Durante el periodo 2005-2008 los resultados de las consultas médicas realizadas en la Unidad Sanitaria de Ticul (Yucatán) por los residentes de la comunidad de San Simón, indicaron que el $36.5 \%$ de los pacientes padecían de insuficiencia respiratoria aguda (IRA) y el $32.1 \%$ de enfermedad diarreica aguda (EDA) (cuadro 1). Aun cuando la incidencia de EDA disminuyó en un $28.22 \%$ desde el 2005 al 2009, posiblemente asociado a la llegada del agua potable, la población más afectada fueron los niños menores de 10 años de los cuales $40 \%$ se encontraban en rango de desnutrición grave y $20 \%$ en grado de desnutrición leve en el momento de la atención. Aun durante el año 2009, el 80\% de los niños que asistieron al Centro de Salud se encontraban con diferentes grados de desnutrición siendo probablemente la causa o la consecuencia de la EDA.

La nutrición adecuada en las distintas etapas de la vida de un individuo es determinante de su salud, de un óptimo desempeño físico e intelectual y por lo tanto de bienestar. Es una regla sin excepción que la desnutrición afecta principalmente al niño menor de seis años por su rápido crecimiento, ya que sus requerimientos nutricionales son más elevados, específicos y difíciles de satisfacer. Una de las causas de la desnutrición, reconocida por la Organización de las Naciones Unidas para la Agricultura y la Alimentación (FAO, por sus siglas en inglés) es la falta de acceso a los alimentos (Burguess y Glasauer, 2004). En México y en especial en Yucatán, el bajo consumo de calcio y proteínas, la enfermedad diarreica aguda (EDA) y la mortalidad infantil preescolar son causa o consecuencia de la desnutrición que padecen muchas comunidades indígenas (Cervera, 1995; Balam, 1996; Ávila-Curiel et al., 1998; Méndez-González, 2010). La imposibilidad de acceder a ciertos productos básicos y complementarios, sobre todo en las localidades marginadas y de difícil acceso, se asocia a la presencia de desnutrición y mala alimentación lo que predispone a patologías. El presente trabajo tiene como objetivo identificar la presencia de factores predisponentes de enfermedad diarreica aguda (EDA) de origen bacteriano en San Simón, Yucatán así como su vulnerabilidad nutricional y ambiental.

\section{METODOLOGÍA}

\subsection{Descripción socio-demográfica de la comunidad de San Simón}

El estudio se llevó a cabo entre julio y agosto del 2009 y la herramienta de evaluación fue una encuesta dirigida a comprender la situación socio-económica de la comunidad. Se efectuó un censo para obtener la información demográfica actualizada entrevistando a un residente mayor de edad en cada una de las 78 casas presentes en la comunidad. De las 78 casas 8 se encontraban vacías durante los meses que se realizó el estudio. La encuesta se integró con 7 áreas de estudio: (1) identificación de la vivienda y del informante, (2) identificación de los integrantes del núcleo familiar (hogares constituidos bajo un mismo techo, parentesco familiar), (3) identificación de la ocupación y presupuesto familiar, (5) identificación de las condiciones de la vivienda (materiales de construcción, ambientes separados y presencia de baño), (6) percepción del estado de salud de los habitantes del hogar, (asistencia a los centros de salud u hospitalizaciones), (7) identificación de hábitos en el consumo de alimentos (\% de alimentos de producción propia y/o adquiridos en puestos cercanos). Se explicó a cada familia acerca de los objetivos y procedimientos del estudio con el auxilio de personal bilingüe, obteniéndose el consentimiento de manera verbal del sujeto a ser encuestado. Este análisis incluyó la encuesta a 70 personas con edades comprendidas entre los 18 y 70 años. El rango de edad de los encuestados fue el siguiente; 28\% entre 18-29 años, 32\% 
entre $29-42$ años, $17 \%$ entre $42-55$ años y $10 \%$ por encima de 55 años. El $5.1 \%$ de los encuestados fueron hombres y $94.8 \%$ mujeres.

Los datos socio-demográficos recabados en este censo se agruparon en 2 indicadores: (a) indicadores de demanda de servicios y (b) indicadores de oferta de servicios para calcular el índice de vulnerabilidad de la comunidad de acuerdo a la metodología propuesta por Cardona y Sarmiento (1990). En los indicadores de demanda (D) se tomaron en cuenta las siguientes características de la población: densidad, población vulnerable, morbilidad, ingreso, nivel de educación, estado nutricio y condiciones de la vivienda. Como indicadores de oferta $(\mathrm{O})$ se tomó en cuenta la presencia de servicios que impactan directa o indirectamente sobre la salud humana y ambiental: la recolección de residuos, agua potable, disponibilidad de alimento, atención médica, apoyo comunitario y vías de comunicación. Cada una de las variables que conformaron el análisis de $\mathrm{D}$ y $\mathrm{O}$ se les asignó un puntaje de 1-5 siendo: 1=óptimo, 3=regular y 5=pésimo. Estos puntajes se sumaron con el fin de obtener el valor acumulado. Para el caso de la oferta, el puntaje varió entre $0-2$ siendo $0=$ inexistente, $1=$ regular y $2=$ óptimo. El índice de vulnerabilidad (V) se calculó con la relación D/O, que de acuerdo a Cardona y Sarmiento (1990) varía entre 1 y 10; considerándose entre 1 a 3 vulnerabilidad baja; entre 4 a 7 vulnerabilidad intermedia y entre 8 a 10 vulnerabilidad alta de sufrir un evento.

\subsection{Evaluación del estado nutricional de los pobladores de la comunidad de San Simón}

La evaluación del estado nutricional de la comunidad se realizó en 106 personas que fueron seleccionadas completamente al azar desde una convocatoria abierta donde se explicaron los objetivos del estudio. El grupo quedó conformado por 33 mujeres, 28 hombres, 24 niñas y 21 niños. Se utilizaron los siguientes indicadores antropométricos: peso (Kg), estatura (m), circunferencia de cintura (CC) y de cadera en adultos $(\mathrm{cm})$; y el perímetro cefálico en niños menores de 3 años de edad. Para la obtención de los indicadores se siguieron los lineamientos establecidos en el manual de la Sociedad Internacional para la Promoción de la Kinantropometria [ISAK, por sus siglas en inglés (2006)], utilizándose una báscula de pedestal con estadímetro $\left(\right.$ Nuevo Leon ${ }^{\circledR}$ ) y una cinta antropométrica (SECA $200^{\circledR}$ ) para la medición de las circunferencias. Las mediciones antropométricas permitieron calcular el índice de masa corporal (IMC=peso / talla) y el índice de cintura-cadera (ICC). Con el IMC se agruparon los individuos de acuerdo a los puntos de corte descritos por Pérez y Marván (2005), siendo IMC $>40=$ obesidad III, IMC 3039.9=obesidad II, IMC 27.1-29.9=obesidad I, IMC 25-27.0=sobrepeso. Los valores normales de IMC son $>18.5 \mathrm{y}<24.9$. Para el indicador ICC se establecieron los siguientes puntos de corte; en hombres $\mathrm{ICC}<0.78=$ ginecoide, ICC $0.78-0.93=$ normal e ICC $>0.93=$ androide; para las mujeres ICC $<0.71=$ ginecoide, ICC $0.71-0.84=$ normal y ICC $>0.84=$ androide. La circunferencia de cintura (CC) se utilizó como indicador de riesgo a la salud (IMSS, 2008). En este caso los puntos de corte fueron: $\mathrm{CC}<80 \mathrm{~cm}$ en la mujer y $94 \mathrm{~cm}$ en el hombre=sin riesgo, $\mathrm{CC} 80-87.9 \mathrm{~cm}$ en la mujer y entre $94-102 \mathrm{~cm}$ en el hombre=riesgo de cardiopatías y diabetes mellitus, $\mathrm{CC}>88 \mathrm{~cm}$ en la mujer y $>102 \mathrm{~cm}$ en el hombre=alto riesgo de enfermedades metabólicas y cardiopatías.

En el caso de los niños se utilizaron los indicadores peso/edad, peso/talla y talla/edad (NOM-031SSA2-1999). Para el indicador peso/edad se utilizaron los siguientes puntos de corte en base a la desviación estándar con relación a la media; $+2 \mathrm{a}+3=$ obesidad, $+1 \mathrm{a}+1.9=$ sobrepeso, $\pm 1=$ peso normal, -1 a $-1.9=$ desnutrición leve, -2 a $-2.9=$ desnutrición moderada, $<3=$ desnutrición grave. En 
cuanto al indicador talla/edad los puntos de corte fueron: +2 a $+3=$ alta, +1 a $+1.9=$ ligeramente alta, $\pm 1=$ estatura normal, -1 a $-1.9=$ ligeramente baja, $<2$ baja. Para al indicador IMC/edad se tomaron los puntos de corte descritos por Mahan y Escott-Stump (2009), siendo; <$3=$ desnutrición severa, $<-2=$ desnutrición, $<-1=$ bajo peso, $\pm 1=$ normal, $>+1=$ sobrepeso, $>+2=$ obesidad.

\subsection{Identificación de vegetales y animales de consumo como portadores de Enterobacterias patógenas}

Con el objetivo de determinar la presencia de Escherichia coli O157:H7 y Salmonella spp. como factores de riesgo, se analizaron 107 muestras por triplicado: 45 muestras de hortalizas y 64 muestras de heces de animales. Para la toma y el manejo de las muestras se siguieron los procedimientos descritos en la Norma Oficial Mexicana NOM-109-SSA1-1994. La determinación de E. coli se realizó a partir de caldo EC (Difco, 231420) incubándose a $44.5 \pm$ $1{ }^{\circ} \mathrm{C}$. Las muestras enriquecidas se sembraron por agotamiento en agar cromogénico (Merck Chromocult, 1.10426.0500). En este medio el sustrato Salmon-GAL se utiliza para la identificación de la enzima $\beta$-D-galactosidasa característica de la bacteria $E$. coli con excepción de la $E$. coli $\mathrm{O} 157$ que es $\beta$-D-glucuronidasa negativa (MUG negativa). Las colonias MUG negativas se sembraron en agar MacConkey sorbitol (Difco, 279100) y se les realizó la prueba de indol utilizando el reactivo de Kovac (p-dimetilamina-benzaldehído, disuelto en butanol-HCL). Finalmente las colonias MUG, sorbitol negativas e indol positivas se confirmaron como E. coli O157:H7 mediante la prueba de aglutinación en látex con anticuerpos monoclonales anti-O157 (Wellcolex, Remel). El resto de las bacterias (MUG positivas) se identificaron utilizando el sistema API-20E (BioMerieux).

La determinación de Salmonella spp. se realizó de acuerdo al protocolo de la NOM-114-SSA11994. El preenriquecimiento se realizó en agua peptonada (AP,0.1\%, pH 7.2\%) incubándose a 37 ${ }^{\circ} \mathrm{C}$ por $24 \mathrm{~h}$. Posteriormente se realizó el enriquecimiento en caldo base tetrationato (Difco, 210430) incubándose a $42.5^{\circ} \mathrm{C}$ por $6 \mathrm{~h}$. El aislamiento selectivo se realizó en agar xilosa lisina desoxicolato (XLD) (BBL, 211836) y agar bilis verde brillante (BVB) (Bioxon, 211708). Luego de $24 \mathrm{~h}$ de incubación a $37{ }^{\circ} \mathrm{C}$, se examinaron las placas para detectar la presencia de colonias típicas de Salmonella. Para la identificación bioquímica se utilizó agar urea (Bioxon 221400), agar hierro lisina (LIA por sus siglas en ingés) (BD/Difco 284920) y agar hierro triple azúcar (TSI por sus siglas en inglés) (BD/Difco 226540). Transcurridas $24 \mathrm{~h}$ de incubación a $37{ }^{\circ} \mathrm{C}$, se consideraron presuntivamente positivas a Salmonella las colonias productoras de ácido sulfhídrico, glucosa positivas y lactosa-sacarosa y urea negativas. Todos los aislamientos de Salmonella fueron confirmados utilizando las pruebas de aglutinación (Wellcolex, Remel).

La presencia de Salmonella spp. y E. coli O157:H7 fue considerada como amenaza y los factores predisponentes para su dispersión en el ambiente y las vías de transmisión al humano como factores de riesgo. Para su valoración se tomó como base la metodología propuesta por Cardona y Sarmiento (1990) asignando valores de 1 a 10; siendo 1 el valor mínimo y 10 el valor máximo para cada uno de los puntos evaluados. El riesgo $(\mathrm{R})$ se calculó multiplicando el índice de vulnerabilidad entre los factores de riesgo. Valores entre 1 y 15 fueron indicativos de riesgo bajo, entre 16 y 35 riesgo intermedio y entre 36 y 100 riesgo alto. 


\section{RESULTADOS}

\subsection{Vulnerabilidad sanitaria-ambiental de la comunidad de San Simón}

San Simón es una comunidad que cuenta con 330 habitantes distribuidos en 78 viviendas. El 25\% de la población es menor de 9 años y un $7.1 \%$ es mayor de 60 años; el 26.9\% se encuentra entre los 9 y 18 años y el $41 \%$ entre 18 a 60 años. Los grupos etarios entre 20 a 59 años de edad conforman la población económicamente activa, con un sueldo promedio de 300 a 1500 pesos quincenales. El género femenino normalmente se encuentra cubriendo las necesidades del hogar y no trabaja por lo que el porcentaje económicamente activo es el $24.4 \%$ de la población. El $37.3 \%$ de la población posee estudios primarios, el $28.2 \%$ estudios secundarios y el $30.9 \%$ no posee estudios. Sus pobladores se dedican a la crianza de cerdos, aves de corral y cultivos de traspatio. Los animales conviven estrechamente con la población, ya sea en las calles o dentro de las casas creando un ambiente de alta contaminación.

Con respecto a la vivienda predomina el tipo de vivienda unifamiliar con paredes de piedra, techo de concreto o de palma y con una sola planta. El 38.8\% de las casas son de piedra y concreto, $24.5 \%$ de cartón, $14.3 \%$ de palma y $13.3 \%$ de madera. El $53 \%$ de las casas poseen cocina con piso de tierra. Las recámaras son por lo general con piso de concreto con excepción del $23 \%$ de las casas que poseen piso de tierra. Utilizan leña como combustible para la preparación de sus alimentos. El $60 \%$ de las casas cuenta con suministro de agua potable que se utiliza fundamentalmente para cocinar. Las casas que no tienen agua potable cocinan con agua de garrafón purificada. En el caso de los baños, el $45 \%$ de las casas cuenta con baños cuyo piso es de concreto, el $10 \%$ de las casas posee letrina y un $15 \%$ no posee baño. A pesar de esto el fecalismo al ras del suelo es una práctica que aún se sigue dando. En las casas que poseen baño $40 \%$ no cuenta con desagüe y solo un $30 \%$ de las casas posee fosa séptica.

Con respecto a los servicios comunitarios, no se cuenta con un transporte especializado en la recolección de desechos. El 94\% de la población incinera la basura o bien la transporta a un centro de acopio a $8 \mathrm{~km}$ del pueblo sobre el camino hacia Uxmal. La comunidad cuenta con escasez de alimentos debido a su estado de marginación. Existen solamente dos tiendas de venta de arroz, frijol, leche en polvo y una variedad de alimentos de baja calidad nutricional en muchas ocasiones caducos. La comunidad se dedica a la crianza de animales y cultivos de traspatio que utilizan como base de su alimentación. Actualmente hay 115 cerdos, 246 gallinas, además de conejos, patos, palomas, perros, gatos y pavos. Un $40 \%$ de las casas producen sus propias hortalizas de consumo y casi el $100 \%$ de las casas utilizan la carne de cerdo y gallinas de su propia crianza como única fuente de proteína en su dieta. Algunos excedentes de producción se venden dentro de la comunidad, principalmente el maíz, calabaza, chile, cebolla y las aves de corral. Los animales no son encerrados en establos o unidades de producción, sino que se encuentran conviviendo con los habitantes en el solar o en zonas comunales. No se cuenta con un rastro para el sacrificio de animales sino que la matanza se realiza en cada solar con ausencia de medidas sanitarias.

En cuanto al estado de salud el $87 \%$ de la población considera que su estado de salud es moderadamente bueno. Cuando padecen algún síntoma de enfermedad el $88 \%$ de la población acude a la medicina tradicional. Esto se debe en parte a la ausencia de un transporte público que 
les permita acceder al Centro de Salud especializado y en otros casos por la falta de un médico fijo en la comunidad. Dentro de las hierbas utilizadas para aliviar trastornos intestinales que cursan con fiebre, el 100\% de los encuestados afirma que utilizan el X'pukin o Palo de chilchaca (Callicarpa acuminata) y cuando el dolor abdominal no cursa con fiebre utilizan una infusión de epazote (Chenopodium ambrosioides).

\subsection{Estado nutricional de la población de San Simón}

Los indicadores antropométricos de las 106 personas de la comunidad de San Simón se presentan en los cuadros 2 y 3 . El $60.6 \%$ de las mujeres y el $53.5 \%$ de los hombres presentaron obesidad y solo el $21.2 \%$ de las mujeres y $32.1 \%$ de los hombres presentaron un relación peso/talla adecuada. La distribución de la grasa corporal fue de tipo androide en $73 \%$ de las mujeres y en $43 \%$ de los hombres. No se presentaron casos de desnutrición en los adultos. Los datos de medición de la circunferencia de cintura (CC) indicó que el $43 \%$ de las mujeres se encuentra con un riesgo muy elevado de desarrollar cardiopatías o enfermedades de índole metabólico.

Para el caso de los niños, el indicador peso/edad demostró que el 58.3\% de las niñas y el 33.3\% de los niños se encuentran en el rango de normalidad, sin embargo se presentaron $29 \%$ de casos de desnutrición grave en las niñas y $14.2 \%$ en los niños. Para el indicador peso/talla el 50\% de los niños se diagnosticó con obesidad. El indicador talla/edad reveló que un $67 \%$ de los niños y niñas son de baja estatura con relación a su edad. Por su parte el indicador de IMC muestra que el 4.5\% de las niñas presenta cuadros de desnutrición severa. En el caso de los niños el $60 \%$ se encuentra por encima de su peso.

\subsection{Presencia de bacterias patógenas como factores de riesgo}

El 80.6\% de los cerdos se diagnosticó como portador de la bacteria Escherichia coli O157:H7. Solo 2 animales fueron portadores del serotipo E. coli O111. En el caso de los cultivos de traspatio el $80.5 \%$ de los cultivos presentó coliformes fecales; el $26.7 \%$ se diagnosticó como $E$. coli, el $11.1 \%$ como Escherichia vulneris y 8.9\% como Escherichia hermanii. En el caso de las aves (patos, palomas, gallinas y pavos) el $42.9 \%$ presentó E. coli, $7.1 \%$ presentó E. hermanii y 3.6\% presentó Escherichia fergusonii. En lo referente a Salmonella, el 28.6\% de las aves fué portador de Salmonella ser. Pullorum y 35.7\% de Salmonella spp. En el caso de los cerdos 5.6\% presentó Salmonella cholerasuis spp. El 8.3\% de las hortalizas dió positivo a Salmonella spp.

\subsection{Estimación de la vulnerabilidad y el riesgo}

La presencia de E. coli O157:H7 en los animales y los cultivos, así como la presencia de Salmonella spp. en el ambiente fuero considerados amenazas. Los indicadores de demanda y oferta del análisis de vulnerabilidad se presentan en el cuadro 4. El valor 4.9 como índice de vulnerabilidad ubica a la comunidad de San Simón con un grado intermedio de padecer enfermedades por Enterobacterias. Sin embargo, de acuerdo a la valoración de los factores predisponentes el riesgo de contaminarse por E. coli O157:H7 y Salmonella spp. es de $39 \pm 10.6$. Este valor ubica a la población de San Simón en un alto nivel de exposición a los agentes causantes de enfermedad diarreica aguda (cuadro 5). 


\section{DISCUSIÓN}

En la triología de las enfermedades de origen ambiental se involucran 3 factores (a) la presencia de agentes patógenos, (b) un ambiente propicio para su trasmisión o diseminación y (c) un hospedero susceptible o vulnerable. De acuerdo al presente estudio estos 3 factores están presentes en la comunidad de San Simón. Los datos del contexto histórico indican que la insuficiencia respiratoria aguda (IRA) y la enfermedad diarreica aguda (EDA) son los principales padecimientos que sufre la comunidad de San Simón (cuadro 1). Los resultados del presente estudio indican que existen las causas que predisponen a éstos padecimientos.

\subsection{Presencia de agentes patógenos}

La valoración de la amenaza situó a la comunidad de San Simón en alto riesgo de contaminarse por Enterobacterias (cuadro 5). La presencia de Escherichia coli O157:H7 en el 80.6\% de los cerdos es considerada una grave amenaza. E. coli O157:H7 es un patógeno enterohemorrágico, que ha sido detectado en las heces de muchos animales incluyendo pollos, corderos, cerdos y animales caseros (Angulo, 1997; Booher et al., 2002). La enfermedad producida por este patógeno se asocia a cuadros de diarrea sanguinolenta y síndrome urémico hemolítico (Caprioli et al., 1994; Bell et al., 1994). Asimismo, la presencia de Salmonella spp. en 35.7\% de las aves puede considerarse otro indicador de riesgo, ya que se vincula a Salmonella enteritidis que se transmite también por animales de crianza y actualmente se asocia a casos de multiresistencia a antibióticos (Feder et al., 2003; Sánchez-Jiménez et al., 2004; Del Pozo et al., 2006). Ambos patógenos son considerados emergentes y su control en salud pública obedece a la severidad de los síntomas (Satcher, 1995; Glynn et al., 1998; Ribot et al., 2002).

El gran número de animales que existe en esta comunidad y las condiciones de crianza fuera de corrales, sobre todo en cerdos, favorece la dispersión de las Enterobacterias contaminando las hortalizas de traspatio (26.7\% a E. coli y $8.3 \%$ a Salmonella spp.). Si bien muchas cepas de $E$. coli no son agentes causales de cuadros graves al humano, se consideran microorganismos índices cuya presencia por encima de ciertos límites de referencia indica la posible presencia de patógenos ecológicamente relacionados. En el caso de las aves infectadas con Salmonella ser. Pullorum y los cerdos infectados con Salmonella cholerasuis spp., estos patógenos causan gastroenteritis en los animales y no se transmiten al hombre, por lo que no se consideran factores de riesgo. Sin embargo, estas especies coexisten con otras especies de Salmonella patógenas para el hombre para las cuales los animales de granja son portadores asintómaticos. Esto significa que los animales pueden liberar durante toda la vida la bacteria al ambiente sin manifestar los síntomas de la enfermedad.

\subsection{Ambiente propicio para transmisión o diseminación del patógeno}

Estudios realizados en el 2001 en Chile, México y Venezuela confirman que los agentes causales de EDA generalmente se trasmiten por vía fecal-oral y adoptan diversas modalidades de acuerdo a las vías de transmisión (Leyva y Mota, 1994). El contexto ambiental de la comunidad no ayuda a la prevención. El fecalismo al ras del suelo, la falta de servicios de saneamiento ambiental, la falta de acorralamiento de los cerdos, la falta de calzado en los niños, el piso de tierra de difícil higiene, aunado al clima propicio de la región favorece la contaminación ambiental y la alta 
posibilidad de trasmisión fecal-oral. A pesar de que la mayoría de las casas tienen construcción de concreto, la cocina es de piso de tierra siendo difícil de sanitizar. Por otro lado a pesar de que el $64.3 \%$ de la población cuenta con baño, no todos tienen fosa y los desechos pasan al aire libre generando contaminación. Estas condiciones propician la contaminación cruzada como una de las principales vías de transmisión de patógenos a los alimentos.

\subsection{Hospedero susceptible o vulnerable}

El análisis de vulnerabilidad indicó que esta comunidad presenta una vulnerabilidad intermedia de padecer una EDA (cuadro 5). Los indicadores de mayor peso para este análisis fueron el estado nutricional de niños y adultos, el porcentaje de morbilidad anual y el bajo nivel de educación e ingresos per cápita.

Las diarreas aunadas al tipo de alimentación afectan el estado de nutrición, ya que durante la mayoría de los cuadros agudos se limita la absorción de los nutrientes en especial, cuando la enfermedad es producida por agentes que lesionan la mucosa intestinal, lo que contribuye a la disminución del aporte calórico. Esto hace que la diarrea sea una entidad patológica concomitante casi siempre en niños desnutridos (Alvarez et al., 2006). Según Valdespino-Gómez (2004), los factores asociados a un mayor riesgo de enfermar e incluso de morir por EDA en Latinoamérica, son la higiene personal deficiente, desnutrición proteico-energética, ausencia o prácticas inapropiadas de lactancia materna, bajo peso al nacimiento, falta de capacitación de la madre para la higiene familiar, contaminación fecal del agua y de alimentos y deficiencia de vitamina A.

De acuerdo a los indicadores de oferta, el aislamiento de la comunidad de los centros urbanos trae consigo carencias en lo referente a alimento, atención médica especializada y servicios para la sanidad ambiental. En esta comunidad la disponibilidad de alimentos es escaza y no en todas las casas se producen cultivos. No se cuenta con un mercado y en la tienda los productos tienen un costo elevado siendo en su mayoría refrescos embotellados y alimentos de bajo valor nutricional. Esto lleva, a que la población no pueda tener una dieta completa con los tres grupos de alimentos, lo que a la larga afecta su estado de nutrición conllevando a casos de obesidad en adultos y desnutrición en niños (Arroyo et al., 2007).

Debido a su estado de nutrición los niños son una población altamente vulnerable y los más expuestos a la amenaza debido al contacto directo con los animales y la tierra. Por otra parte, ellos dependen de otras personas para su alimentación, quienes a veces no tienen los recursos necesarios. Como conclusión del trabajo cabe mencionar la urgencia de una precautoria intervención en la comunidad de San Simón orientada a disminuir los factores de riesgo de enfermedades producidas por un mal saneamiento ambiental. El acorralamiento de los cerdos, la mejora de la dieta y de las condiciones del hogar son puntos a mejorar. Según Fuentes-Díaz et al., (2008) el 70\% de las muertes por diarrea puede evitarse si se realiza una adecuada promoción y prevención con respecto a los factores de riesgo antes señalados, a través de acciones educativas y de promoción de la salud. Esto mejoraría paulatinamente la condición de salud de los niños y sus familias permitiendo un desarrollo digno. 


\section{BIBLIOGRAFÍA}

Álvarez, J.B., Quintero, S., Villero, S. (2006). Factores de riesgo en el estado nutricional por la enfermedad diarreica aguda. Revista Colombiana de pediatría. Consultado en http://www.encolombia.com/medicina/pediatria/pediatria38303-factores $2 . h t m$ [marzo 2011].

Angulo, F.J. (1997). Multidrug resistant Salmonella typhimurium definitive type DT104. Emerg Infect Dis. 3, 414-22.

Arroyo, P., Fernández, V., Loría, A., Pardío, J., Laviada, H.1., Vargas-Ancona. L., Ward, R. (2007). Obesidad, morfología corporal y presión arterial en grupos urbanos y rurales de Yucatán. Salud Pública de México, 49, 274-285.

Ávila-Curiel, A., Shamah-Levy, T., Galindo-Gómez, G., Rodríguez-Hernández, G. BarragánHeredia, L. (1998). La desnutrición infantil en el medio rural mexicano. Salud Pública de México, 40,150-160.

Balam, G. (1996). La frecuencia de la desnutrición y sus factores socioeconómicos en Yucatán. Avance y perspectiva, 6,197-206.

Bell, B.P., Goldoft, M., Griffin, P., Davis, M.A., Gordon, D.C., Tarr, P. (1994). A multistate outbreak of Escherichia coli O157:H7-associated bloody diarrhea and hemolytic uremic syndrome from hamburgers: the Washington experience. JAMA, 272,1349-53.

Booher, S.L., Cornick, N.A., Moon, H.W. (2002). Persistence of Escherichia coli O157:H7 in experimentally infected swine. Vet. Microbiol. 2002, 89, 69-81.

Burguess, A., Glasauer, P. (2004) Family Nutrition Guide. Eds. Ann Burgess y Peter Glasauer. FAO Corporate Document Repository. http://www.fao.org/docrep/007/y5740e/y5740e03.htm

Caprioli, A., Luzzi, I., Rosmini, F., Resti, C., Edefonti, A., Perfumo, F., Farina, C., Goglio, A., Gianviti, A., Rizzoni, G. (1994). Community wide outbreak of hemolytic-uremic syndrome associated with non-O157 verocytoxin-producing Escherichia coli. J. Infect. Dis. 169, 208-11.

Cardona, O.D., Sarmiento, J.P. (1990). Análisis de Vulnerabilidad y Evaluación del Riesgo para la Salud de una Población expuesta a desastres. Colombian Red Cross Society (CRC).

Cervera, M.D., Murguia, R.E., Méndez, R.M., UC, L. (1995). Estado nutricional de la población menor de tres años de dos regiones socio-económicas del sur de Yucatán. En: López, S. y Serrano, C. (Eds.). Búsquedas y Hallazgos. Estudios Antropológicos en Homenaje a Johanna Faulhaber, IIA-UNAM, D.F, México. 
Coneval. Consejo Nacional de Evaluación de la Política de Desarrollo Social. (2005). Mapas de pobreza por ingresos y rezago social. Consejo Nacional de Evaluación de la Política de Desarrollo Social, D.F., México.

Del Pozo, L., Silva, N., Valencia, A., Soto, J., Riveros, J.C., Sacsaquispe, R., Calderón, R., Suárez, V. (2006). Estudio de un brote intrahospitalario por Salmonella typhimurium productora de beta-lactamasa de espectro extendido SHV-5. Universidad Nacional Mayor de San Marcos. Anales de la Facultad de Medicina, 67, 318-366.

Feder, I.E., Wallace, M., Gray, J.T., Fratamico, P., Fedorka-Cray, J., Pearce, R., Call, J.E., Perrine, R., Luchansky, J.B. (2003). Isolation of Escherichia coli O157:H7 from intact colon fecal samples of swine. Emerg. Infect. Dis., 9, 380-383.

Fuentes-Díaz, Z., Rodríguez-Salazar, O., Salazar-Diez, M., Rodríguez-Hernández, O. (2008). Factores de riesgo de las enfermedades diarreicas agudas en menores de cinco años. Revista Archivo Médico de Camaguey. AMC(Online) vol.12, n.2.

Glynn, M.K., Bopp, C., Dewitt, W., Dabney, P., Mokhtar, M., Angulo, F.J. (1998). Emergence of multidrug-resistant Salmonella enterica serotype typhimurium DT104 infections in the United States. N. Eng. J. Med., 338,1333-1338.

IMSS. Instituto Mexicano del Seguro Social. (2008). Circunferencia de la cintura, 2008. [consultado 2010 enero 12 . Consultado http://www.imss.gob.mx/salud/Nutricion/Circunferencia_cintura.htm [enero, 2011].

ISAK. International Standards for Anthropometric Assessment. (2006). (A manual for teaching materials for accreditation.) ( $2^{\text {nd }}$ Ed.). Potchefstroom University for CHE, SAF: ISAK.

Le Minor, L. (1992). The genus Salmonella. In The Prokaryotes. Vol. III. Ed. Balows, A., Trüper, H. G., Dworkin, M., Harder, W., and Schleifer, K.-H. New York.

Leyva, L.S., Mota, H.F. (1994). Manual de consulta educativa en diarrea. Ed. Med Hosp. Infant. Mex., D.F., México.

Mahan, L., Escott-Stump, S. (2009). Krause Dietoterapia. 12a Ed.. Elsevier Masson, Barcelona.

Méndez-González, R. (2010). Condiciones de vida y salud en zonas indígenas de Yucatán, México: 1990 y 2005. Población y Salud en Mesoamérica vol. 8. Consultado en http://www.redalyc.uaemex.mx/src/inicio/ArtPdfRed.jsp?iCV $\mathrm{ve}=44613827003$. [marzo 2011].

NOM-031-SSA2-1999. Norma Oficial Mexicana (1999). Para la Atención a la Salud del Niño.

NOM-109-SSA1-1994. Norma Oficial Mexicana (1994). Procedimientos para la toma, Manejo y Transporte de Muestras de Alimentos para su Análisis Microbiológico. 
NOM114-SSA1-1994. Norma Oficial Mexicana (1994). Bienes y Servicios. Método para la determinación de Salmonella en alimentos.

Pérez, A.B., Marvan, L. (2005). Manual de dietas normales y terapéuticas: Los alimentos en la salud y la enfermedad. 5a edición. Editorial La Prensa Médica Mexicana, D.F., México.

Ribot, E.M., Wierzba, R.K., Angulo, F.J., Barrett, T.J. (2002). Salmonella enterica serotype typhimurium DT104 isolated from humans, United States, 1985, 1990, and 1995. Emerg. Infect. Dis. 8, 387-391.

Sánchez-Jiménez, M.J., Caraballo-Guzmán, A.J., Cardona-Castro, N.M., Bernal-Parra, C., TuliaZapata, C., Durango, H.E. (2004). Determinación del perfil de sensibilidad y resistencia a antibióticos seleccionados, en cepas de Salmonella spp. Aisladas en Antioquia durante los años 2002 y 2003. Revista CES MEDICINA, 18, 35-42.

Satcher, D. (1995). Emerging infections: getting ahead of the curve. Emerg. Infect. Dis.,1,1-6.

Valdespino-Gómez, A. (2004). Epidemiology and etiology of infectious diarrhea. Rev Latinoam. Microbiol., 36, 307-24.

Yates, M. (1992). Biomonitors of environmental contamination. Encyclopedia of Microbiology. Vol 1, Academic Press, Inc, New York. 


\section{AGRADECIMIENTOS}

Agradecemos a la comunidad de San Simón por su noble participación en el estudio. Un especial agradecimiento para los maestros Fernando Carrillo Sosa, Director de la Telesecundaria "Vicente Guerrero" y Felipe Chan, Director de la Escuela Primaria "18 de Marzo", ambos de la comunidad de San Simón por el apoyo logístico incondicional. Este estudio fue financiado por el Fondo Mixto de fomento a la Investigación Científica y Tecnológica CONACYT-Gobierno del Estado de Yucatán (2008,06-108103). 
Cuadro 1. Casos clínicos atendidos en los pobladores de la comunidad de San Simón durante el periodo 2005-2009.

\begin{tabular}{lcc}
\hline Síntoma o Enfermedad & $\mathbf{N}^{\mathbf{0}}$ de casos & \% de casos \\
\hline IRAS* & 318 & 36.5 \\
Presión arterial & 5 & 0.6 \\
EDAS** & 280 & 32.1 \\
Fiebre & 42 & 4.8 \\
Dolor abdominal & 74 & 8.5 \\
Vómitos & 24 & 2.8 \\
Diabetes & 8 & 0.9 \\
Piedra & 3 & 0.3 \\
Reuma & 20 & 2.3 \\
Anemia & 5 & 0.6 \\
Arritmias & 3 & 0.3 \\
Alergias & 3 & 0.3 \\
Dolor lumbar & 3 & 0.3 \\
Cefaleas & 52 & 6.0 \\
Amigdalitis & 7 & 0.8 \\
Gripe & 12 & 1.4 \\
Otitis & 5 & 0.6 \\
Conjuntivitis & 8 & 0.9 \\
\hline $\mathrm{N}^{\mathrm{o}}$ de casos atendidos & 872 & \\
$\mathrm{~N}^{\mathrm{o}}$ de casos/año & 174 & \\
$\mathrm{~N}^{\mathrm{o}}$ enfermos/habitante/año & 0.6 & \\
\hline
\end{tabular}

* Insuficiencia respiratoria aguda

** Enfermedad diarreica aguda 
Cuadro 2. Indicadores antropométricos del estado nutricional de la población adulta de la comunidad de San Simón.

\begin{tabular}{|c|c|c|}
\hline \multirow[t]{2}{*}{ Indicador } & \multicolumn{2}{|c|}{ IMC (\%) } \\
\hline & Mujeres & Hombres \\
\hline Obesidad III & 15.2 & 0.0 \\
\hline Obesidad II & 30.3 & 32.1 \\
\hline Obesidad I & 15.2 & 21.4 \\
\hline Sobrepeso & 18.2 & 14.3 \\
\hline Normalidad & 21.2 & 32.1 \\
\hline Desnutrición I & 0.0 & 0.0 \\
\hline Desnutrición II & 0.0 & 0.0 \\
\hline Desnutrición III & 0.0 & 0.0 \\
\hline \multirow[t]{2}{*}{ Indicador } & \multicolumn{2}{|c|}{ ICC (\%) } \\
\hline & Mujeres & Hombres \\
\hline Ginecoide & 0 & 0 \\
\hline Androide & 72.2 & 46.4 \\
\hline Normal & 27.3 & 53.6 \\
\hline \multirow[t]{2}{*}{ Indicador } & \multicolumn{2}{|c|}{ CC (\%) } \\
\hline & Mujeres & Hombres \\
\hline Sin riesgo & 21.2 & 60.7 \\
\hline Riesgo & 36.4 & 21.4 \\
\hline Alto riesgo & 42.4 & 15.9 \\
\hline
\end{tabular}

$\mathrm{IMC}=$ Índice de masa corporal

$\mathrm{ICC}=$ Índice de cintura cadera

$\mathrm{CC}=$ Circunferencia de cintura 
Cuadro 3. Indicadores antropométricos del estado nutricional de los niños en la comunidad de San Simón.

\begin{tabular}{|c|c|c|c|c|c|}
\hline \multirow[t]{2}{*}{ Indicador } & \multicolumn{2}{|c|}{ Peso/Estatura (\%) } & \multirow[t]{2}{*}{ Indicador } & \multicolumn{2}{|c|}{ Peso/Edad (\%) } \\
\hline & Niñas & Niños & & Niñas & Niños \\
\hline Obesidad & 0.0 & 50.0 & Obesidad & 0.0 & 14.2 \\
\hline Sobrepeso & 20.0 & 33.3 & Sobrepeso & 8.3 & 4.7 \\
\hline Normal & 60.0 & 16.7 & Normal & 58.3 & 33.3 \\
\hline Desnutrición leve & 0.0 & 0.0 & Desnutrición leve & 4.2 & 19.0 \\
\hline Desnutrición & & & Desnutrición & & \\
\hline $\begin{array}{l}\text { moderada } \\
\text { Desnutrición orave }\end{array}$ & $\begin{array}{c}20.0 \\
00\end{array}$ & $\begin{array}{l}0.0 \\
0.0\end{array}$ & $\begin{array}{l}\text { moderada } \\
\text { Desnutrición orave }\end{array}$ & $\begin{array}{c}0.0 \\
292\end{array}$ & $\begin{array}{c}9.5 \\
14.2\end{array}$ \\
\hline Desnutricion grave & & 0.0 & & 29.2 & \\
\hline \multirow[t]{2}{*}{ Indicador } & \multicolumn{2}{|c|}{ IMC/Edad (\%) } & Indicador & \multicolumn{2}{|c|}{ Estatura/Edad (\%) } \\
\hline & Niñas & Niños & & Niñas & Niños \\
\hline Obesidad & 22.7 & 30.0 & Alta & 4.16 & 0 \\
\hline Sobrepeso & 13.6 & 30.0 & Ligeramente alta & 0 & 0 \\
\hline Normal & 54.5 & 15.0 & Normal & 16.66 & 19 \\
\hline Bajo peso & 0.0 & 25.0 & Ligeramente baja & 12.50 & 14.28 \\
\hline Desnutrición & 4.5 & 0.0 & Baja & 66.66 & 66.66 \\
\hline Desnutrición severa & 4.5 & 0.0 & & & \\
\hline
\end{tabular}

*IMC=Índice de masa corporal 
Cuadro 4. Análisis del índice de vulnerabilidad de la comunidad de San Simón en base a indicadores de oferta y demanda de salud.

\begin{tabular}{|c|c|c|}
\hline Indicadores demanda & Valor & Justificación \\
\hline Densidad poblacional & 1 & Poco densa \\
\hline Estructura según edad & 3 & $25 \%$ de la población niños - grupo vulnerable \\
\hline Estructura de salud morbilidi & 4 & 0.6 enfermos/habitantes/año \\
\hline Ingreso económico familiar & 5 & Muy bajo \\
\hline Tipo de casa habitación & 3 & $53 \%$ cocina piso tierra y $23 \%$ recámara piso tierra \\
\hline Baños & 4 & $45 \%$ de la población cuentan con baños \\
\hline Educación & 4 & $30.9 \%$ analfabetismo \\
\hline Estado nutricional niños & 5 & $\begin{array}{l}29 \% \text { desnutrición niñas } \\
14.2 \% \text { desnutrición niños } \\
4.5 \% \text { niñas desnutrición severa } \\
50 \% \text { obesidad en niños }\end{array}$ \\
\hline Estado nutricional adultos & 5 & $\begin{array}{l}60.6 \% \text { mujeres obesidad } \\
53.5 \% \text { hombres obesidad } \\
73 \% \text { mujeres conformación androide } \\
40 \% \text { hombres conformación androide }\end{array}$ \\
\hline \multicolumn{3}{|l|}{ Indicadores de oferta } \\
\hline Servicios de salud & 1 & 1 centro asistencial \\
\hline Atención médica & 1 & No permanente \\
\hline Apoyo comunitario & 2 & Permanente \\
\hline Vías de comunicación & 0 & Muy escasas \\
\hline Recolección residuos & 0 & No hay transporte \\
\hline Agua potable & 1 & $60 \%$ agua potable \\
\hline Disponibilidad de alimento & 1 & De propia crianza, no hay alimentos procesados \\
\hline Combustible & 1 & Leña para cocinar \\
\hline $\begin{array}{l}\text { Rastro para sacrificio } \\
\text { animales }\end{array}$ & 0 & Ausente \\
\hline Índice de vulnerabilidad & 4.9 & MODERADA \\
\hline
\end{tabular}




\section{Cuadro 5. Valoración de factores de riesgo de la comunidad de San Simón.}

\begin{tabular}{lcc}
\hline Amenaza & Valoración & \\
\hline Presencia de E. coli O157:H7 en el ambiente & 10 & \\
Presencia de Salmonella spp. en el ambiente & 10 & Riesgo \\
\hline Factores de riesgo & Valoración & 41 \\
\hline Falta de acorralamiento de animales & 9 & 45 \\
\hline Presencia de patógenos en alimentos consumo & 10 & 45 \\
\hline Presencia animales portadores de patógenos & 10 & 41 \\
\hline Fecalismo al ambiente & 9 & 36 \\
\hline No uso de calzado & 8 & 23 \\
\hline Piso tierra difícil higiene & 5 & 23 \\
\hline Climática vientos & 5 & $\mathbf{3 9 \pm 1 0 . 6}$ \\
\hline Nivel de riesgo ALTO & & \\
\hline
\end{tabular}

